\title{
Factors associated with HIV-related stigma toward people living with HIV among nurses in Liangshan Yi Autonomous Prefecture, China: a cross-sectional study
}

\section{Yao Yin}

Sichuan University West China Hospital

\section{Angela Chia-Chen Chen}

Arizona State University

\section{Shaoping Wan}

Sichuan Cancer Hospital and Research Institute Hong Chen ( $\sim 1366109878 @ q q . c o m$ )

https://orcid.org/0000-0002-1867-1107

\section{Research article}

Keywords: HIV-related stigma, HIV/AIDS, Nurses, China

Posted Date: July 18th, 2019

DOl: https://doi.org/10.21203/rs.2.11578/v1

License: (c) (i) This work is licensed under a Creative Commons Attribution 4.0 International License. Read Full License

Version of Record: A version of this preprint was published at Frontiers in Psychiatry on August 23rd, 2021. See the published version at https://doi.org/10.3389/fpsyt.2021.714597. 


\section{Abstract}

Background The Liangshan Yi Autonomous Prefecture has one of the most serious human immunodeficiency virus (HIV) epidemics in China. Evidence shows HIV-related stigma toward people living with HIV (PLWH) among nurses impedes HIV prevention and treatment. However, only limited research about HIV-related stigma toward PLWH from the perspective of nurses in Liangshan has been conducted. This cross-sectional study aimed to assess HIV-related stigma toward PLWH among nurses and determine factors associated with it in Liangshan, China. Methods Using a stratified, random cluster sampling method, registered nurses $(N=1,248$; primary hospitals $=102$, secondary hospitals $=592$, tertiary hospitals $=554$ ) were recruited 10 hospitals in Liangshan. All participants completed an anonymous online survey measuring sociodemographic characteristics, HIV-related stigma and HIV knowledge. We used multiple stepwise regression analysis to examine factors associated with HIV-related stigma toward PLWH among these nurses. Results The mean score of HIV-related stigma among nurses was 50.7 (SD =

8.3; range 25-78). Nurses who were more experienced, had higher levels of education, and were working in tertiary hospitals reported higher level of HIV-related stigma. Those who had better HIV knowledge, reported a willingness to receive HIV-related training, were working in areas that had a high prevalence of HIV, had prior experience working in acquired immune deficiency syndrome (AIDS) specialized hospitals, and worked in hospitals that had policies to protect PLWH showed a lower level of HIV-related stigma toward PLWH. Conclusions Findings suggested that providing culturally congruent education and training about HIV and care, and having hospitals that promoted policies protecting PLWH, may reduce HIVrelated stigma toward PLWH among nurses in China.

\section{Background}

Stigma related to the human immunodeficiency virus (HIV) is prevalent and has been identified to be a major barrier for HIV prevention and treatment [1]. Researchers suggest that HIV-related stigma is also still a barrier for people living with HIV (PLWH) to disclose their HIV status and to seek and adhere to treatments; consequently, this barrier has an adverse impact on their health[2-4]. HIV-related stigma exists in nursing care worldwide and has large impact on the health outcomes of PLWH [5-9].

Herek [10] defined HIV-related stigma as "prejudice, discounting, discrediting, and discrimination directed at people perceived to have acquired immune deficiency syndrome (AIDS) or HIV" (p.1107).

Manifestations of HIV-related stigma within the healthcare system include neglecting and avoidance of nursing care, exaggerated precautions, judgmental remarks and behavior, labelling patients and viewing them as attention seekers and breaches of confidentiality [9, 10][11-14]. Empirical evidence shows that the main causes of HIV-related stigma among healthcare providers are their lack of awareness of the stigma perceived by PLWH and the negative impact of stigma on PLWH, fear of casual contact due to poor knowledge about HIV transmission and the association of HIV with improper or immoral behavior [15]. 
Researchers have found that certain sociodemographic characteristics of nurses, such as age, gender, race, marital status, educational level and certain religious belief are associated with HIV-related stigma toward PWLH [16-21]. Furthermore, longer working experience, lower levels of HIV knowledge and nurses working at Islamic hospitals have also been associated with higher levels of stigmatizing attitudes $[5,17,20,22]$.

Findings about the relationship of working at HIV clinics with the level of HIV-related stigma among healthcare providers are mixed in the literature $[11,19,23]$. Structural and policy support in HIV clinics have been found to reduce HIV-related stigma toward PLWH in healthcare providers, including HIV-related training, resources for universal precaution and available access to post-exposure prophylaxis $[11,15]$.

Due to the rapid increase of HIV epidemic in China, Chinese government announced a national AIDS control policy-“Four Frees and One Care"-in 2003. This national policy refers to free antiretroviral drugs treatment to AIDS patients living in rural areas or individuals without insurance living in urban areas, free voluntary counseling and testing (VCT), free drugs treatment to HIV-infected pregnant women to prevent mother-to-child transmission (PMCT) and HIV testing of newborns, free schooling for AIDS orphans, and care and financial assistance to PLWH [24]. Under this national policy, a growing number of PLWH have received free tests, consultations, and treatments, and the associated mortality rate has been reduced [24].

In China, the HIV epidemic is charaterized as low national prevalence (0.055\%) [25]. By the end of 2017, the reported number of PLWH in China was 758,610 [25]. However, China has the second distribution of new HIV infections in Asia and the Pacific region [26]. The Liangshan Yi Autonomous Prefecture is unique; it has the largest concentration of $\mathrm{Yi}(\mathrm{G})$, one of the largest minority groups in China, and has one of the most serious epidemics of HIV in China [27]. In fact, nearly 50\% of PLWH in Liangshan were infected through injecting drugs [28]. Its special cultural background, extreme poverty, low level of education, and prevalent substance use through shared needles in this geographic area have made $\mathrm{Yi}$ people particularly vulnerable to HIV infection [29]. As a shortage of human resources and financial burden, nurses have expanding roles in providing HIV-related care,such as providing consultaion, education and follow-up visits [30]. However, there is a very little understanding of nurses' HIV-related stigma toward PLWH and factors associated with the stigma in Liangshan. To address this gap, our study aimed to answer the following research questions: (a) what is the status of HIV-related stigma toward PLWH among nurses in Liangshan, and (b) what are the factors associated with HIV-related stigma toward PLWH in nurses in Liangshan?

\section{Methods}

\section{Study design and participants}

We conducted a cross-sectional study from September to November 2017 in Liangshan. Hospitals in China are designated as primary, secondary and tertiary hospitals based on a hospital's capacity of providing medical care, education and research. Primary hospitals have less than 100 beds and focus on 
focus on health education, disease prevention and health care, rehabilitation, family planning, and common and frequent disease management. Secondary hospitals usually have beds between 100 and 500 and provide comprehensive health care, medical education and research. Tertiary hospitals typically have more than 500 beds and provide comprehensive health services at the city, provincial or national level and carry a more important role in medical education and research [31].

Using a stratified, random cluster sampling method, our study sites included 3 primary, 5 secondary and 2 tertiary hospitals in Liangshan. Our target participants were registered nurses aged 18 or older who had worked in the selected hospitals for at least 6 months and consented to participate.

\section{Data Collection}

In each hospital, we first contacted the head nurse of its nursing department for approval and assistance in recruiting nurses. Once we received approval, we sent eligible nurses an online survey link, including a consent form describing the purpose, procedures, the potential risks and benefits of the study. Nurses who consented to participate received a link to access the online anonymous survey.

\section{Variables and Instruments}

Our survey consisted of variables shown in the literature to be related to HIV-related stigma in healthcare providers. It included questions about individual and workplace characteristics, HIV-related stigma scale for health care workers [32], and HIV knowledge scale[33]. Both the stigma and knowledge scales have been tested in Chinese population [32,33].

\section{Individual and workplace characteristics}

Individual characteristics included age, gender, ethnicity, educational level, years of experience working as a nurse, experience of providing care to PLWH, prior experience of HIV-related training, and willingness to receive HIV-related training. Workplace characteristics consisted of questions regarding level of hospital s/he worked, HIV prevalence area (high vs. low; high prevalence was defined as an area with $>1 \%$ HIV prevalence), prior experience working in AIDS specialized hospitals, universal precaution supplies and policies in workplace that protect PLWH from stigma.

\section{HIV-related stigma}

We used a 17-item "HIV-related stigma scale for health care workers" developed by Stein and Li [32] to assess the HIV-related stigma among nurses. This multidimensional scale includes 5 subscales:

Discrimination Intent at Work (4 items; e.g., you would be willing to work with HIV positive patients), Opinion about Health Care for HIV-infected Patients ( 3 items; e.g., people who got infected with HIV/AIDS through drug use deserve good quality medical care), Prejudiced Attitudes (4 items; e.g., people who got HIV/AIDS through sex and drug use got what they deserved), Internalized Shame (3 items; e.g., if you worked with HIV positive patients, you would feel embarrassed to tell other people about it), and Fear of PLWHA (3 items; e.g., you feel afraid of PLWH). Scores for each item ranges from 1 (strongly agree) to 5 
(strongly disagree). Some items were reverse-scored (e.g., the Opinion about Health Care for HIV-infected Patients subscale) when appropriate, and a higher total score suggested higher levels of HIV-related stigma toward PLWH. Stein and Li [32] reported adequate internal consistency (alpha $=0.68-0.82)$ and construct validity of the scale.

\section{HIV knowledge}

We measured HIV knowledge using a Chinese version HIV knowledge scale [33] adapted from Jemmott and colleagues' work [34]. It is a 24-item scale assessing nurses' knowledge of basic characteristic and transmission routes of HIV. Responses of each scale item were: "true", "false" or "not sure". Participants received 1 point if their answers were correct, and they received 0 points if their answers were incorrect or "not sure." A higher total score indicated higher levels of HIV knowledge.

\section{Statistical analyses}

We used IBM SPSS 22.0 [35] to manage and analyze data. We used the mean, standard deviation ( $S D$ ), frequency and percentage to describe distributions of variables. Pearson's $r$ correlations, t-tests and oneway analyses of variance (ANOVA) were used to examine relationships between key variables and HIVrelated stigma toward PWLH. We further conducted multiple stepwise regression analysis to investigate factors associated with HIV-related stigma toward PLWH. A p-value less than 0.05 was used to indicate statistical significance.

Power estimate: We calculated the required sample size based on the formula below [36] The required study size was 1,225 ( $\mathrm{S}=8.92$; effective size $=0.50 ; \mathrm{a}=0.05)$.

$$
\mathrm{n}=\left(\frac{Z_{\alpha / 2} \cdot \mathrm{S}}{\delta}\right)^{2}
$$

\section{Results}

A total of 1,256 registered nurses participated in this study. Eight of them were excluded because they were younger than 18 years old or had less than 6 months of work experience. Thus, a final sample of 1,248 participants was included in the analysis.

\section{Individual and workplace characteristics}

As shown in Table 1, the mean age of nurses was 30.2 years old (SD=7.2), with a range of 19-55. Most (98.4\%) of them were females; $72.2 \%(n=902)$ were Han ethnicity; $62.8 \%(n=784)$ had an associated degree; and $90.4 \%(n=1,128)$ worked in low prevalence areas. In terms of working experience, the mean year was $8.8(S D=7.8)$. Regarding HIV-related experience and training, although only $12.7 \%(n=158)$ of 
the participants had prior experience working in AIDS specialized hospitals, $84.2 \%$ ( $n=1,051)$ of them had provided care for PLWH. While $90.1 \%(n=1,124)$ of the participants were willing to receive HIVrelated training, only $63.1 \%(n=787)$ of them had received the training in the past. Majority $(95.5 \%)$ of the participants reported that the hospitals where they worked provided sufficient universal precaution supplies, and $86.9 \%(n=1,084)$ reported that the hospitals had policies to protect PLWH from stigma.

As for HIV knowledge, the mean total score was $16.3(\mathrm{SD}=2.9)$. The mean total score for HIV-related stigma among registered nurses was $50.7(\mathrm{SD}=8.3)$. Among the 5 subscales, "prejudiced attitudes" had the highest scores $(M=3.7, S D=0.7)$ while "discrimination intent at work" had the lowest scores $(M=$ $2.3, \mathrm{SD}=0.6)$.

\section{Results of univariate analysis}

The relationships between HIV-related stigma and other study variables are shown in Table 2. A higher HIV-related stigma score was significantly related to longer years of work experience, and lower levels of HIV knowledge. The results of group differences in HIV-related stigma score are presented in Table 3. Nurses who were members of the Han ethnic group, were working in low HIV prevalence areas, had higher educational levels (i.e., associate or higher vs. Diploma), had longer years of work experience, worked at a higher hospital level (Tertiary vs. Secondary vs. Primary), and had not worked in specialized AIDS hospitals reported higher HIV-related stigma score. Nurses' willingness to receive HIV-related training, experience of having received HIV-related training in the past, and working at a hospital where had policies to protect PLWH from stigma reported a lower level of HIV-related stigma toward PLWH.

\section{Findings of multiple stepwise regression analysis}

Table 4 presents the multiple stepwise regression analysis results. The findings suggested that a higher HIV-related stigma was associated with nurses who were more educated (associated degree or higher vs. diploma), had longer years of working experience, were working in the tertiary hospitals, did not have prior experience working in specialized AIDS hospitals, were not willing to receive HIV-related training, worked in low HIV prevalence areas, working in a hospital that had policies to protect PLWH from stigma and had higher levels of HIV knowledge.

All the selected variables accounted for $14.0 \%$ of the variance (R2). Among the selected variables, HIV knowledge had the strongest effect to explain HIV-related stigma toward PLWH among nurses (Beta=-0.227, $\mathrm{P}<.001$ ).

\section{Discussion}

To the best of our knowledge, this study is the first aimed at examing HIV-related stigma toward PLWH and factors associated with it among nurses in Liangshan, China. The mean score for HIV-related stigma was high, which is similar to findings from research conducted in other regions of China [36,37]. As high levels of HIV-related stigma toward vulnerable PLWH population are related poor nursing care and 
negative health outcomes [38,39], PLWH may not receive proper nursing care due to nurses' high levels of HIV-related stigma toward this population in Liangshan.

Individual characteristics. Compared with nurses who had an associate degree or higher level of education, diploma-educated nurses reported a lower level of HIV-related stigma, consistent with prior research $[40,41]$. Deacon and Boulle [42] suggested that higher-educated nurses might have more prejudicial attitudes due to a greater gap in social status between them and PLWH. Similar to other researchers $[9,21,43]$, we found that nurses with better HIV knowledge reported no matter what the level of education, a lower level of HIV-related stigma toward PWLH overall, and in particularly, less fear of AIDS. This finding suggested the critical role of HIV knowledge in reducing fear-based stigma [44]. Since there was no significant difference of HIV knowledge between nurses with different educational levels, HIV education should target all nurses.

We also found that nurses who had longer years of working experience reported a higher level of HIVrelated stigma toward PLWH, a finding similar to that in other studies [17,22]. A possible explanation is that nurses who were more experienced might assign nursing care to nurses with less experience [14]. Thus, junior nurses were more likely to provide care to PLWH and this experience might reduce their levels of HIV-related stigma compared with nurses who were more experienced.

Workplace characteristics. Consistent with Li et al [40], we found that nurses working in the tertiary hospitals had a higher level of HIV-related stigma toward PLWH. In China, tertiary hospitals that provide comprehensive services to a larger patient population are more likely to receive referrals of PLWH with opportunistic infections or other illnesses that might not be able to be cured. The complexities of providing care to terminally ill AIDS patients may make these nurses experience burnout and form avoidance attitudes $[5,45]$.

Our findings supported that nurses working in high HIV prevalence areas and having prior working experience in AIDS specialized hospitals had a lower level of HIV-related stigma, which is also similar to findings in prior research [46,47]. Nurses who worked in high HIV prevalence areas and had prior working experience in AIDS specialized hospitals had more experience in taking care of PLWH. Increasing exposure to and more experience of interacting with PLWH were found to be related to a lower level of HIV-related stigma and higher confidence in taking care of this vulnerable population $[23,48]$. Moreover, nurses working in high HIV prevalence areas and having prior working experience in AIDS specialized hospitals received more institutional supports and training [46,47], which were found to reduce their anxiety about HIV infection, thereby reducing their stigma toward PLWH $[19,38,40]$.

The significant relationship between nurses' prior HIV-related training and their HIV-related stigma was indicated in t-test but not in regression analyses when other variables were held constant. This inconsistent finding may be due to the differences in HIV training, such as content, format and frequency, as they have significant impact on the effectiveness of the training. Further, nurses who were willing to receive HIV-related training reported lower levels of HIV-related stigma. Thus, future research should 
examine characteristics of successful HIV training for nurses to reduce HIV-related stigma toward PWLH and promote PWLH's optimal health outcomes.

Research has supported the conclusion that stigma-reduction interventions, including policy development, led to positive changes in HIV-related stigma in health care settings [49], which is consistent with our findings. The results indicated that nurses working in the hospitals that implemented policies to protect PLWH from stigma had lower levels of HIV-related stigma toward PLWH. However, we did not find a significant relationship between hospitals that provided sufficient universal precaution supplies and nurses' HIV-related stigma. This might be explained by a lack of variance in this variable, as the majority $(95.5 \%)$ of nurses reported that universal precaution supplies were provided in the hospitals where they worked.

Given the high prevalence rate of PLWH in Liangshan, providing nurses with comprehensive HIV-related training and requiring hospitals to have policies to reduce HIV-related stigma toward PWLH are critical to reducing the high prevalence rate of PLWH.

Limitations. Several study limitations should be noted. Given the nature of cross-sectional survey design, it is challenging to draw causal relationships between variables. Further, HIV-related stigma is a sensitive topic in China, and so the validity of self-reported data may not be optimal. With these limitations in mind, our anonymous survey should have minimized the participants' social desirability and bias. Using the stratified random cluster sampling to obtain a powered sample with sufficient sample size enhanced the representation of our target population and therefore increased the external validity of the findings.

\section{Conclusions}

The present study was the first to examine the level of HIV-related sigma toward PWLH and factors associated with the stigma in nurses in Liangshan, an area that has one of the most serious HIV epidemics in China. The findings revealed high levels of HIV-related stigma among nurses in Liangshan. Nurses' high levels of HIV knowledge, willingness to receive HIV-related training, and working in the hospitals that had anti-stigma policies were significantly related to their lower levels of stigma toward PWLH. Comprehensive HIV-related training that addresses these factors and incorporates the unique economic and cultural contexts of Liangshan will help nurses to reduce associated stigma and, in turn, provide culturally congruent care to PWLH and promote their optimal health outcomes.

\section{Abbreviations}

HIV: human immunodeficiency virus; PLWH: people living with HIV; AIDS: acquired immune deficiency syndrome; VCT: voluntary counseling and testing; PMCT: prevent mother-to-child transmission; SD: standard deviation; ANOVA: analyses of variance; SEE, standard error of estimation.

\section{Declarations}




\section{Ethics approval and consent to participate}

We received approval from the West China Hospital Medical Ethics Committee prior to implementation the study (No. 430[2017]). The electronic version of informed consent was received from all participants.

\section{Consent for publication}

Not applicable.

\section{Availability of data and materials}

The datasets generated and/or analysed during the current study are not publicly available due to policy restriction.

\section{Competing interests}

The authors declare that they have no competing interests.

\section{Funding}

They study received no supporting of any funding.

\section{Authors' contributions}

The study was designed by YY, ACC, SW and HC. Data collection was performed by YY, SW and HC. YY and ACC conducted data analysis and data interpretation. YY developed first draft of the article. YY, ACC, $\mathrm{SW}$ and $\mathrm{HC}$ contributed to revisions of manuscript for important intellectual content. All authors read and approved the final manuscript.

\section{Acknowledgements}

We would like to express our sincere gratitude to the nurse who participated in this research and to hospital administrators who approved and assisted with recruitment.

\section{References}

1. Joint United Nations Programme on HIV/AIDS (UNAIDS). Global AIDS update 2016. Available at: http://www.unaids.org/sites/default/files/media_asset/global-AIDS-update-2016_en.pdf

2. Brinsdon A, Abel G, Desrosiers J. "I'm taking control": How people living with HIV/AIDS manage stigma in health interactions. AIDS Care 2017; 29:185-188.

3. Essomba EN, Kollo B, Ngambi MK, et al. Stigma and discrimination associated with HIV/AIDS in health care settings: A comparative study in two hospitals of different categories in DoualaCameroon. Journal of Medical \& Biomedical Sciences 2017;3: 14-22. 
4. Saki M, Kermanshahi SMK, Mohammadi E, Mohraz M. Perception of patients with HIV/AIDS from stigma and discrimination. Iranian Red Crescent Medical Journal 2015;17: e23638.

5. Delobelle P, Rawlinson JL, Ntuli S, Malatsi I, Decock R, Depoorter AM. HIV/AIDS knowledge, attitudes, practices and perceptions of rural nurses in south Africa. Journal of Advanced Nursing 2009; 65:1061-1073.

6. Kermode M, Holmes W, Langkham B, Thomas MS, Gifford S. HIV-related knowledge, attitudes \& risk perception amongst nurses, doctors \& other healthcare workers in rural India. Indian Journal of Medical Research 2005;122: 258-264.

7. Li L, Li Y, Zeng K, Wu Y. Knowledge and attitudes to HIV/AIDS in Chinese registered nurses. Journal of Central South University (Medical Science) 2011; 36: 121-127.

8. Vorasane S, Jimba M, Kikuchi K, et al. An investigation of stigmatizing attitudes towards people living with HIV/AIDS by doctors and nurses in Vientiane, Lao PDR. BMC Health Services Research 2017;17: 125 .

9. Wu HC, Ko NY, Shih CC, Feng MC. HIV/AIDS: An exploration of the knowledge, attitude, infection risk perceptions, and willingness to care of nurses. Hu Li Za Zhi 2014; 61: 43-53.

10. Herek GM. AIDS and stigma. American Behavioral Scientist, 1999; 42: 1106-1116.

11. Andrewin A, Chien LY. Stigmatization of patients with HIV/AIDS among doctors and nurses in Belize. AIDS Patient Care and STDs 2008; 22:897-906.

12. Greeff M, Phetlhu R, Makoae LN, et al. Disclosure of HIV status: Experiences and perceptions of persons living with HIV/AIDS and nurses involved in their care in Africa. Qualitative Health Research 2008;18: 311-324.

13. Kohi TW, Makoae L, Chirwa M, et al. HIV and AIDS stigma violates human rights in five African countries. Nursing Ethics 2006; 13: 404-415.

14. Manganye BS, Maluleke TX, Lebese RT. Professional nurses' views regarding stigma and discrimination in the care of HIV and AIDS patients in rural hospitals of the Limpopo province, South Africa. African Journal of AIDS Research 2013; 12: 33-40.

15. Nyblade L, Stangl A, Weiss E, Ashburn K. Combating HIV stigma in health care settings: What works? Journal of the International AIDS Society 2009;12: 15.

16. Aghamolaei T, Tavafian SS, Hasani L, Zare S. Attitudes of healthcare providers towards patients with HIV/AIDS in Bandar Abbas. Archives of Iranian Medicine 2009; 12: 298-301.

17. Kostak MA, Unsar S, Kurt S, Erol O. Attitudes of turkish midwives and nurses working at hospitals towards people living with human immunodeficiency virus/acquired immune deficiency syndrome. International Journal of Nursing Practice 2012;18: 437-444.

18. Ekstrand ML, Ramakrishna J, Bharat S, Heylen E. Prevalence and drivers of HIV stigma among health providers in urban India: Implications for interventions. Journal of the International AIDS Society 2013;16: 18717. 
19. Stringer KL, Turan B, Mccormick L, et al. HIV-related stigma among healthcare providers in the Deep South. AIDS and Behavior 2016; 20: 115-125.

20. Waluyo A, Culbert GJ, Levy J, Norr KF. Understanding HIV-related stigma among Indonesian nurses. The Journal of the Association of Nurses in AIDS Care 2015; 26: 69-80.

21. Zarei N, Joulaei H, Darabi E, Fararouei M. Stigmatized attitude of healthcare providers: A barrier for delivering health services to HIV positive patients. International journal of community based nursing and midwifery 2015; 3(4): 292-300.

22. Suominen T, Koponen N, Mockiene V, et al. Nurses' knowledge and attitudes to HIV/AIDS - an international comparison between Finland, Estonia and Lithuania. International Journal of Nursing Practice 2010; 16:138-147.

23. Feyissa GT, Abebe L, Girma E, Woldie M. Stigma and discrimination against people living with HIV by healthcare providers, Southwest Ethiopia. BMC Public Health 2012;12:522.

24. Ministry of Foreign Affairs People's Republic of China (MFA). Report on China's implementation of the millennium development goals (2000-2015). Available at: http://www.undp.org/content/dam/china/docs/Publications/UNDP-CH-SSC-MDG2015_English.pdf

25. UNAIDS. Delivering as one UN The UN Joint Programme on AIDS in China 2018-2019. Available at: http://www.unaids.org.cn/pics/20181128163211.pdf

26. UNAIDS. Ending AIDS: Progress towards the 90-90-90 targets. Available at: http://www.unaids.org/sites/default/files/media_asset/Global_AIDS_update_2017_en.pdf

27. National Health Commission of the People's Republic of China (NHC). 2015 China AIDS response progress report. Available at: http://www.unaids.org/sites/default/files/country/documents/CHN_narrative_report_2015.pdf

28. Mo SD, Wang Z, Deng F. Analysis of epidemilogical characteristics and countermeaure of AIDS in Liangshan state. Modern Prevention Medicine 2008;35: 630-635.

29. Zhu C, Zhou Y, Song X, Jiang Q. Review on the influene factors of AIDS epidemic and control in Liangshan Prefecture Sichuan Province of China. Fudan University Journal of Medical Sciences 2015; 42: 675-679.

30. Chen WT, Shiu CS, Simoni J, Fredriksen-Goldsen K, Zhang F, Zhao H. Optimizing HIV care by expanding the nursing role: Patient and provider perspectives. Journal of Advanced Nursing 2010; 66: 260-268.

31. World Health Organization (WHO). People's Repuclic of China health system review. Available at: http://apps.who.int/iris/bitstream/handle/10665/208229/9789290617280_eng.pdf? sequence $=1$ \&isAllowed $=y$

32. Stein JA, Li L. Measuring HIV-related stigma among Chinese service providers: Confirmatory factor analysis of a multidimensional scale. AIDS and Behavior 2008; 12:789-795.

33. Wang $H$, Yang A, Williams AB. Knowledge, attitudes, willingness of care about HIV/AIDS among nursing college students. Journal Nursing Science 2003;18:166-168. 
34. Jemmott LS, Jemmott JB, Cruzcollins M. Predicting AIDS patient care intentions among nursing students. Nursing Research 1992; 41:172-177.

35. IBM Corp. Released 2013. IBM SPSS Statistics for Windows, Version 22.0. Armonk, NY: IBM Corp.

36. Yang J, Liu H, Niu L. Research on correlation between nurses' discrimination and their nursing behaviors for HIV infections and AIDS patients. Chinese Nursing Research 2015; 29:2448-2452.

37. Han Y. The study of correlation between nurses' stigma toward PLWHA and nurse caring behaviors. Unpublished master's thesis 2009; Central South University, Changsha.

38. Mill J, Harrowing J, Rae T, et al. Stigma in AIDS nursing care in sub-Saharan Africa and the Caribbean. Qualitative Health Research 2013; 23: 1066-1078.

39. Kay ES, Rice WS, Crockett KB, Atkins GC, Batey DS, Turan B. Experienced HIV-Related stigma in health care and community settings: Mediated associations with psychosocial and health outcomes. Journal of Acquired Immune Deficiency Syndromes 2018;77: 257-263.

40. Li L, Wu Z, Wu S, Zhaoc Y, Jia M, Yan Z. HIV-related stigma in health care settings: A survey of service providers in China. AIDS Patient Care and STDs 2007; 21: 753-762.

41. Vyas KJ, Patel GR, Shukla D, Mathews WC. A comparison in HIV-associated stigma among healthcare workers in urban and rural Gujarat. SAHARA Journal 2010; 7: 71-75.

42. Deacon H, Boulle A: Commentary. Factors affecting HIV/AIDS-related stigma and discrimination by medical professionals. International Journal of Epidemiology 2007; 36: 185-186.

43. Li L, Li Y, Zeng K, Wu Y. Knowledge and attitudes to HIV/AIDS in Chinese registered nurses. Journal of Central South University (Medical Science) 2011; 36: 121-127.

44. Abdelrahman I, Lohiniva AL, Kandeel A, et al. Learning about barriers to care for people living with HIV in Egypt: A qualitative exploratory study. Journal of the International Association of Providers of AIDS Care 2015; 14: 141-147.

45. Roomaney R, Steenkamp J, Kagee A. Predictors of burnout among HIV nurses in the Western Cape. Curationis 2017;40:e1-e9.

46. Hamama L, Tartakovsky E, Eroshina K, et al. Nurses' job satisfaction and attitudes towards people living with HIV/AIDS in Russia. International Nursing Review 2014; 61: 131-139.

47. Li L, Lin C, Wu Z, Comulada WS, Ding Y Regional differences in HIV prevalence and individual attitudes among service providers in China. Social Science \& Medicine 2012; 75: 283-287.

48. Salih MH, Tessema GA, Cherkos EA, Ferede AJ, Anlay DZ. Stigma towards people living on HIV/AIDS and associated factors among nurses' working in Amhara region referral hospitals, Northwest Ethiopia: A cross-Sectional study. Advances in Nursing 2017; 2017: 1-7.

49. Pulerwitz J, Oanh KTH, Akinwolemiwa D, Ashburn K, Nyblade L. Improving hospital-based quality of care by reducing HIV-related stigma: Evaluation results from Vietnam. AIDS and Behavior 2015;19: 246-256.

\section{Tables}


Table 1 Individual and workplace characteristics, HIV knowledge and HIV-related stigma toward PLWH 


\begin{tabular}{|c|c|c|c|c|c|}
\hline Variables & $\mathrm{N}$ & $\%$ & Mean & $\mathrm{SD}$ & Range \\
\hline Age (years) & & & 30.2 & 7.2 & $19-55$ \\
\hline \multicolumn{6}{|l|}{ Gender } \\
\hline Male & 20 & 1.6 & & & \\
\hline Female & 1228 & 98.4 & & & \\
\hline \multicolumn{6}{|l|}{ Ethnicity } \\
\hline Han & 902 & 72.2 & & & \\
\hline Minority & 346 & 27.8 & & & \\
\hline \multicolumn{6}{|l|}{ Educational level } \\
\hline Diploma & 92 & 7.4 & & & \\
\hline Associate degree & 784 & 62.8 & & & \\
\hline Baccalaureate degree or above & 372 & 29.8 & & & \\
\hline Years of working experience & & & 8.8 & 7.8 & $0.5-37$ \\
\hline \multicolumn{6}{|l|}{ Provide care for PLWH } \\
\hline Yes & 1051 & 84.2 & & & \\
\hline No & 197 & 15.8 & & & \\
\hline \multicolumn{6}{|c|}{ Willingness to receive HIV-related training } \\
\hline Yes & 1124 & 90.1 & & & \\
\hline No & 124 & 9.9 & & & \\
\hline \multicolumn{6}{|l|}{ Prior HIV-related training } \\
\hline Yes & 787 & 63.1 & & & \\
\hline No & 461 & 36.9 & & & \\
\hline \multicolumn{6}{|l|}{ Level of hospital } \\
\hline Primary hospital & 102 & 8.2 & & & \\
\hline Secondary hospital & 592 & 47.4 & & & \\
\hline Tertiary hospital & 554 & 44.4 & & & \\
\hline \multicolumn{6}{|l|}{ HIV prevalence area } \\
\hline High prevalence areas (>1\%) & 120 & 9.6 & & & \\
\hline Low prevalence areas (<1\%) & 1128 & 90.4 & & & \\
\hline
\end{tabular}


Sufficient universal precaution supplies

Yes

No

Policies to protect PLWH from stigma

Yes

No or doubt

HIV knowledge

HIV-related stigma scale

Prejudiced attitudes subscale

Fear of PLWHA subscale

Opinion about health care for HIV/AIDS patients subscale

Internalized shame subscale

Discrimination intent at work subscale
$1192 \quad 95.5$

$56 \quad 4.5$

$1084 \quad 86.9$

$164 \quad 13.1$

$16.3 \quad 2.9 \quad 7-23$

$50.7 \quad 8.3 \quad 25-78$

$\begin{array}{lll}3.7 & 0.7 & 1-5\end{array}$

$\begin{array}{lll}3.2 & 0.8 & 1-5\end{array}$

$3.1 \quad 0.8 \quad 1-5$

$2.7 \quad 0.7 \quad 1-5$

$2.3 \quad 0.6 \quad 1-4.8$

\section{PLWH people living with HIV; $S D$ standard deviation}

Table 2 Correlations between age, years of working experience, HIV knowledge and HIV-related stigma

\begin{tabular}{lllll}
\hline Variables & 1 & 2 & 3 & 4 \\
\hline 1 Age(years) & 1.000 & - & - & - \\
2 Years of working experience & $0.951^{* * *}$ & 1.000 & - & - \\
3 HIV knowledge & $0.165^{* *}$ & $0.156^{* *}$ & 1.000 & - \\
4 HIV-related stigma & 0.052 & $0.062^{*}$ & $-0.228^{* *}$ & 1.000 \\
\hline
\end{tabular}

* $P<0.05$

** $P<0.01$ 
Table 3 Differences in HIV-related stigma by individual and workplace characteristics a

\begin{tabular}{|c|c|c|c|}
\hline \multirow[t]{2}{*}{ Variables } & \multicolumn{2}{|c|}{ Stigma score } & \multirow[t]{2}{*}{ Statistical test } \\
\hline & Mean & $\mathrm{SD}$ & \\
\hline Ethnicity & & & $\mathrm{t}=3.702^{* *}$ \\
\hline Han & 51.2 & 8.4 & \\
\hline Minority & 49.3 & 7.9 & \\
\hline Educational level & & & $\mathrm{F}=5.886^{*}$ \\
\hline Diploma & 48.0 & 7.7 & \\
\hline Associate degree & 50.7 & 8.2 & \\
\hline Baccalaureate degree or above & 51.3 & 8.5 & \\
\hline Willingness to receive HIV-related training & & & $\mathrm{t}=6.213^{* *}$ \\
\hline Yes & 50.2 & 8.2 & \\
\hline No & 55.0 & 8.1 & \\
\hline Prior HIV-related training & & & $\mathrm{t}=2.645^{*}$ \\
\hline Yes & 50.2 & 8.2 & \\
\hline No & 51.5 & 8.4 & \\
\hline Level of hospital & & & $\mathrm{F}=11.848^{* *}$ \\
\hline Primary hospital & 48.3 & 9.8 & \\
\hline Secondary hospital & 50.0 & 8.1 & \\
\hline Tertiary hospital & 51.8 & 8.1 & \\
\hline HIV prevalence area & & & $\mathrm{t}=6.058 * *$ \\
\hline High prevalence areas & 47.0 & 6.9 & \\
\hline Low prevalence areas & 51.1 & 8.3 & \\
\hline Prior experience working in AIDS specialized hospitals & & & $\mathrm{t}=4.618^{* *}$ \\
\hline Yes & 47.8 & 7.4 & \\
\hline No & 51.1 & 8.4 & \\
\hline Policies to protect PLWH from stigma & & & $t=-5.695 * *$ \\
\hline Yes & 50.2 & 8.2 & \\
\hline No or doubt & 54.1 & 8.1 & \\
\hline
\end{tabular}




\section{PLWH people living with HIV; SD standard deviation}

a Only significant results have been listed

$* P<0.05$

** $P<0.01$

Table 4 Multiple stepwise regression analysis of variables in relation to HIV-related stigma

\begin{tabular}{|c|c|c|c|c|c|}
\hline Variables & $\mathrm{B}$ & SEE & $\beta$ & $\mathrm{t}$ & $P$-value \\
\hline Educational level & 0.951 & 0.402 & 0.065 & 2.367 & 0.018 \\
\hline Years of working experience (years) & 0.095 & 0.029 & 0.089 & 3.326 & 0.001 \\
\hline \multicolumn{6}{|c|}{ Willingness to receive HIV-related training (ref: yes) } \\
\hline No & 4.022 & 0.736 & 0.145 & 5.467 & $<0.001$ \\
\hline \multicolumn{6}{|l|}{ Level of hospital (ref: tertiary hospital) } \\
\hline Primary hospital & -2.635 & 0.837 & -0.087 & -3.150 & 0.002 \\
\hline Secondary hospital & -1.225 & 0.483 & -0.074 & -2.536 & 0.011 \\
\hline \multicolumn{6}{|c|}{ HIV prevalence area (ref: low prevalence areas) } \\
\hline High prevalence areas & -2.561 & 0.797 & -0.091 & -3.211 & 0.001 \\
\hline \multicolumn{6}{|c|}{ Prior experience working in AIDS specialized hospitals (ref: no) } \\
\hline Yes & -2.268 & 0.666 & -0.091 & -3.404 & 0.001 \\
\hline \multicolumn{6}{|c|}{ Policy to protect PLWH from stigma (ref: yes) } \\
\hline No & 3.028 & 0.651 & 0.123 & 4.649 & $<0.001$ \\
\hline HIV knowledge & -0.662 & 0.078 & -0.227 & -8.449 & $<0.001$ \\
\hline
\end{tabular}

$S E E$ standard error of estimation 